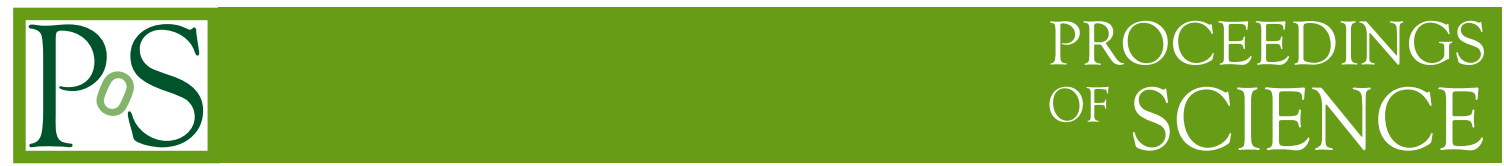

\title{
$\rho$ meson decay from the lattice
}

\section{CP-PACS collaboration:}

\section{S. Aoki, ${ }^{a, b}$ M. Fukugita, ${ }^{c}$ K.-I. Ishikawa, ${ }^{d}$ N. Ishizuka*, ${ }^{a, e}{ }^{a}$ K. Kanaya, ${ }^{a}$ Y. Kuramashi, ${ }^{a, e}$} Y. Namekawa, ${ }^{f}$ M. Okawa, ${ }^{d}$ K. Sasaki, ${ }^{e}$ A. Ukawa, ${ }^{a, e}$ T. Yoshié ${ }^{a, e}$

${ }^{a}$ Graduate School of Pure and Applied Sciences, University of Tsukuba, Tsukuba, Ibaraki 305-8571, Japan

${ }^{b}$ Riken BNL Research Center, Brookhaven National Laboratory, Upton, New York 11973, USA

${ }^{c}$ Institute for Cosmic Ray Research, University of Tokyo, Kashiwa 277-8582, Japan

${ }^{d}$ Department of Physics, Hiroshima University, Higashi-Hiroshima, Hiroshima 739-8526, Japan

${ }^{e}$ Center for Computational Sciences, University of Tsukuba, Tsukuba, Ibaraki 305-8577, Japan

${ }^{f}$ Department of Physics, Nagoya University, Nagoya 464-8602, Japan

We present preliminary results on the $\rho$ meson decay width estimated from the scattering phase shift of the $I=1$ two-pion system. The phase shift is calculated by the finite size formula for nonzero total momentum frame (the moving frame) derived by Rummukainen and Gottlieb, using the $N_{f}=2$ improved Wilson fermion action at $m_{\pi} / m_{\rho}=0.41$ and $L=2.53 \mathrm{fm}$.

XXIVth International Symposium on Lattice Field Theory

July 23-28, 2006

Tucson, Arizona, USA

\footnotetext{
${ }^{*}$ Speaker.

†E-mail : ishizuka@ccs.tsukuba.ac.jp
} 


\section{Introduction}

Lattice study of the $\rho$ meson decay is an important step for understanding of the dynamical aspect of hadron reactions induced by strong interactions. There are already three studies $[1,2,3]$. The earlier two studies employed the quenched approximation ignores the decay into two ghost pions. The most recent work, while using the $N_{f}=2$ dynamical configurations, concentrated on the $\rho \rightarrow \pi \pi$ transition amplitude rather than the full matrix including the $\rho \rightarrow \rho$ and $\pi \pi \rightarrow \pi \pi$ amplitudes. All three studies were carried out at an unphysical kinematics $m_{\pi} / m_{\rho}>1 / 2$.

In this work we attempt to carry out a more rigorous approach. We estimate the decay width from the scattering phase shift for the $I=1$ two-pion system. The finite size formula presented by Rummukainen and Gottlieb [4] is employed for an estimation of the phase shift. Calculations are carried out with $N_{f}=2$ full QCD configuration previously generated for a study of light hadron spectrum with a renormalization group improved gauge action and a clover fermion action at $\beta=$ $1.8, \kappa=0.14705$ on a $12^{3} \times 24$ lattice [5]. The parameters determined from the spectrum analysis are $1 / a=0.92 \mathrm{GeV}, m_{\pi} / m_{\rho}=0.41$, and $L=2.53 \mathrm{fm}$. All calculations of this work are carried out on VPP5000/80 at the Academic Computing and Communications Center of University of Tsukuba.

\section{Method}

In order to realize a kinematics such that the energy of the two-pion state is close to the resonance energy $m_{\rho}$, we consider the non-zero total momentum frame (the moving frame) [4] with the total momentum $\mathbf{p}=2 \pi / L \cdot \mathbf{e}_{3}$. The initial $\rho$ meson is assigned a polarization vector parallel to $\mathbf{p}$. One of the final two pions carry the momentum $\mathbf{p}$, while the other pion is at rest. The energies ignoring hadron interactions are then given by $E_{1}^{0}=\sqrt{m_{\pi}^{2}+p^{2}}+m_{\pi}$ for the two-pion state and $E_{2}^{0}=\sqrt{m_{\rho}^{2}+p^{2}}$ for the $\rho$ meson. We neglect higher energy states whose energies are much higher than $E_{1}^{0}$ and $E_{2}^{0}$. On our full QCD configurations, the invariant mass for the two-pion state takes $\sqrt{s}=0.97 \times m_{\rho}$, while $1.47 \times m_{\rho}$ is expected for the zero total momentum. The $\rho$ meson at zero momentum cannot decay energetically, so that it can be used to extract $m_{\rho}$.

The hadron interactions shift the energy from $E_{n}^{0}$ to $E_{n}(n=1,2)$. These energies $E_{n}$ are related to the two-pion scattering phase shift $\delta(\sqrt{s})$ through the Rummukainen-Gottlieb formula [4], which is an extension of the Lüscher formula [6] to the moving frame. The formula for the total momentum $\mathbf{p}=p \mathbf{e}_{3}$ and the $\mathbf{A}_{2}^{-}$representation of the rotation group on the lattice reads

$$
\frac{1}{\tan \delta(\sqrt{s})}=\frac{1}{2 \pi^{2} q \gamma} \sum_{\mathbf{r} \in \Gamma} \frac{1+\left(3 r_{3}^{2}-r^{2}\right) / q^{2}}{r^{2}-q^{2}}
$$

where $\sqrt{s}=\sqrt{E^{2}-p^{2}}$ is the invariant mass, $k$ is the scattering momentum $\left(\sqrt{s}=2 \sqrt{m_{\pi}^{2}+k^{2}}\right), \gamma$ is the Lorentz boost factor $(\gamma=E / \sqrt{s})$, and $q=k L /(2 \pi)$. The summation for $\mathbf{r}$ in (2.1) runs over the set

$$
\Gamma=\left\{\mathbf{r} \mid r_{1}=n_{1}, r_{2}=n_{2}, r_{3}=\left(n_{3}+\frac{p}{2} \frac{L}{2 \pi}\right) / \gamma, \mathbf{n} \in \mathbf{Z}^{3}\right\} .
$$

The right hand side of (2.1) can be evaluated by the method described in Ref. [7]. 
In order to calculate $E_{1}$ and $E_{2}$ we construct a $2 \times 2$ matrix time correlation function,

$$
G(t)=\left(\begin{array}{ll}
\left\langle 0\left|(\pi \pi)^{\dagger}(t)(\pi \pi)\left(t_{s}\right)\right| 0\right\rangle & \left\langle 0\left|(\pi \pi)^{\dagger}(t) \rho_{3}\left(t_{s}\right)\right| 0\right\rangle \\
\left\langle 0\left|\rho_{3}^{\dagger}(t)(\pi \pi)\left(t_{s}\right)\right| 0\right\rangle & \left\langle 0\left|\rho_{3}^{\dagger}(t) \rho_{3}\left(t_{s}\right)\right| 0\right\rangle
\end{array}\right) .
$$

Here, $\rho_{3}(t)$ is an interpolating operator for the neutral $\rho$ meson with the momentum $\mathbf{p}=2 \pi / L \cdot \mathbf{e}_{3}$ and the polarization vector parallel to $\mathbf{p} ;(\pi \pi)(t)$ is an interpolating operator for the two pions given by

$$
(\pi \pi)(t)=\frac{1}{\sqrt{2}}\left(\pi^{-}(\mathbf{p}, t) \pi^{+}(\mathbf{0}, t)-\pi^{+}(\mathbf{p}, t) \pi^{-}(\mathbf{0}, t)\right),
$$

which belongs to the $\mathbf{A}_{2}^{-}$and iso-spin representation with $I=1, I_{z}=0$.

We can extract the two energy eigenvalues by a single exponential fitting of the two eigenvalues $\lambda_{1}\left(t, t_{R}\right)$ and $\lambda_{2}\left(t, t_{R}\right)$ of the normalized matrix $M\left(t, t_{R}\right)=G(t) G^{-1}\left(t_{R}\right)$ with some reference time $t_{R}[8]$ assuming that the lower two states dominate the correlation function.

In order to construct the meson state with non-zero momentum we introduce a $U(1)$ noise $\xi_{j}(\mathbf{x})$ in three-dimensional space whose property is

$$
\frac{1}{N_{R}} \sum_{j=1}^{N_{R}} \xi_{j}^{\dagger}(\mathbf{x}) \xi_{j}(\mathbf{y})=\delta^{3}(\mathbf{x}-\mathbf{y}) \quad \text { for } N_{R} \rightarrow \infty .
$$

We calculate the quark propagator

$$
Q\left(\mathbf{x}, t \mid \mathbf{q}, t_{s}, \xi_{j}\right)=\sum_{\mathbf{y}}\left(D^{-1}\right)\left(\mathbf{x}, t ; \mathbf{y}, t_{s}\right) \cdot\left[\mathrm{e}^{i \mathbf{q} \cdot \mathbf{y}} \xi_{j}(\mathbf{y})\right],
$$

regarding the term in the square bracket as the source. The two point function of the meson with the spin content $\Gamma$ and the momentum $\mathbf{p}$ can be constructed from $Q$ as

$$
\frac{1}{N_{R}} \sum_{j=1}^{N_{R}} \sum_{\mathbf{x}} \mathrm{e}^{-i \mathbf{p} \cdot \mathbf{x}} \cdot\left\langle\gamma_{5} Q^{\dagger}\left(\mathbf{x}, t \mid \mathbf{0}, t_{s}, \xi_{j}\right) \gamma_{5} \Gamma^{\dagger} Q\left(\mathbf{x}, t \mid \mathbf{p}, t_{s}, \xi_{j}\right) \Gamma\right\rangle,
$$

where the bracket refers to the trace for color and spin indeces.

The quark contraction for the $\pi \pi \rightarrow \pi \pi$ and the $\pi \pi \rightarrow \rho$ components of $G(t)$ are given by

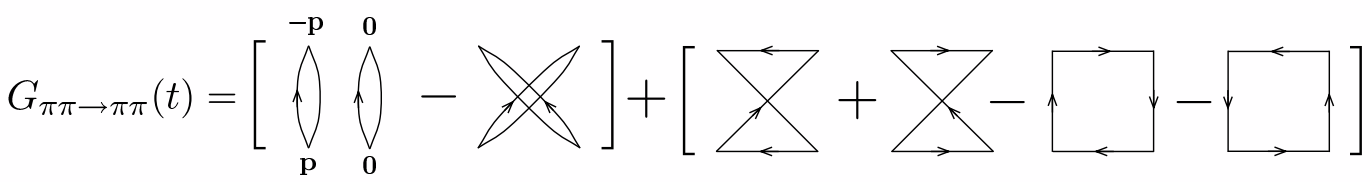

$$
\begin{aligned}
& G_{\pi \pi \rightarrow \rho}(t)=\left[\bigwedge_{\mathbf{p}}^{-\mathrm{p}}-\bigwedge_{0}\right]
\end{aligned}
$$

where the four verteces for the $\pi \pi \rightarrow \pi \pi$ and three verteces for the $\pi \pi \rightarrow \rho$ components refer to the pion or the $\rho$ meson with definite momentum. The time direction is upward in the diagrams, and the $\rho \rightarrow \pi \pi$ component is given by changing the time direction. 
The first term of the $\pi \pi \rightarrow \pi \pi$ component in (2.8) can be calculated by introducing another $U(1)$ noise $\eta_{j}(\mathbf{x})$ having the same property as $\xi_{j}(\mathbf{x})$ in (2.5);

$$
\frac{1}{N_{R}} \sum_{j=1}^{N_{R}} \sum_{\mathbf{x}, \mathbf{y}} \mathrm{e}^{-i \mathbf{p} \cdot \mathbf{x}} \cdot\left\langle Q^{\dagger}\left(\mathbf{x}, t \mid \mathbf{0}, t_{s}, \xi_{j}\right) Q\left(\mathbf{x}, t \mid \mathbf{p}, t_{s}, \xi_{j}\right)\right\rangle\left\langle Q^{\dagger}\left(\mathbf{y}, t \mid \mathbf{0}, t_{s}, \eta_{j}\right) Q\left(\mathbf{y}, t \mid \mathbf{0}, t_{s}, \eta_{j}\right)\right\rangle .
$$

The second term of (2.8) is obtained by exchanging the momentum of the sink in (2.9).

In order to construct the other terms of (2.8) we calculate a quark propagator of another type by the source method,

$$
W\left(\mathbf{x}, t\left|\mathbf{k}, t_{1}\right| \mathbf{q}, t_{s}, \xi_{j}\right)=\sum_{\mathbf{z}}\left(D^{-1}\right)\left(\mathbf{x}, t ; \mathbf{z}, t_{1}\right) \cdot\left[\mathrm{e}^{i \mathbf{k} \cdot \mathbf{z}} \gamma_{5} Q\left(\mathbf{z}, t_{1} \mid \mathbf{q}, t_{s}, \xi_{j}\right)\right],
$$

where the term in the square bracket is regarded as the source in solving the propagator. Using $W$ we can construct the third to sixth terms in the $\pi \pi \rightarrow \pi \pi$ component of (2.8) by

$$
\begin{aligned}
& \text { 3rd }=\frac{1}{N_{R}} \sum_{j=1}^{N_{R}} \sum_{\mathbf{x}} \mathrm{e}^{-i \mathbf{p} \cdot \mathbf{x}} \cdot\left\langle W^{\dagger}\left(\mathbf{x}, t\left|\mathbf{0}, t_{s}\right|-\mathbf{p}, t_{s}, \xi_{j}\right) W\left(\mathbf{x}, t|\mathbf{0}, t| \mathbf{0}, t_{s}, \xi_{j}\right)\right\rangle, \\
& \text { 4th }=\frac{1}{N_{R}} \sum_{j=1}^{N_{R}} \sum_{\mathbf{x}} \mathrm{e}^{-i \mathbf{p} \cdot \mathbf{x}} \cdot\left\langle W\left(\mathbf{x}, t\left|\mathbf{0}, t_{s}\right| \mathbf{p}, t_{s}, \xi_{j}\right) W^{\dagger}\left(\mathbf{x}, t|\mathbf{0}, t| \mathbf{0}, t_{s}, \xi_{j}\right)\right\rangle, \\
& \text { 5th }=\frac{1}{N_{R}} \sum_{j=1}^{N_{R}} \sum_{\mathbf{x}} \mathrm{e}^{-i \mathbf{p} \cdot \mathbf{x}} \cdot\left\langle W\left(\mathbf{x}, t\left|\mathbf{p}, t_{s}\right| \mathbf{0}, t_{s}, \xi_{j}\right) W^{\dagger}\left(\mathbf{x}, t|\mathbf{0}, t| \mathbf{0}, t_{s}, \xi_{j}\right)\right\rangle, \\
& \text { 6th }=\frac{1}{N_{R}} \sum_{j=1}^{N_{R}} \sum_{\mathbf{x}} \mathrm{e}^{-i \mathbf{p} \cdot \mathbf{x}} \cdot\left\langle W^{\dagger}\left(\mathbf{x}, t\left|-\mathbf{p}, t_{s}\right| \mathbf{0}, t_{s}, \xi_{j}\right) W\left(\mathbf{x}, t|\mathbf{0}, t| \mathbf{0}, t_{s}, \xi_{j}\right)\right\rangle .
\end{aligned}
$$

The two terms of $\pi \pi \rightarrow \rho$ of (2.8) can be similarly constructed by

$$
\begin{aligned}
& 1 \text { st }=\frac{1}{N_{R}} \sum_{j=1}^{N_{R}} \sum_{\mathbf{x}} \mathrm{e}^{-i \mathbf{p} \cdot \mathbf{x}} \cdot\left\langle W^{\dagger}\left(\mathbf{x}, t\left|-\mathbf{p}, t_{s}\right| \mathbf{0}, t_{s}, \xi_{j}\right)\left(\gamma_{5} \gamma_{3}\right) Q\left(\mathbf{x}, t \mid \mathbf{0}, t_{s}, \xi_{j}\right)\right\rangle, \\
& \text { 2nd }=\frac{1}{N_{R}} \sum_{j=1}^{N_{R}} \sum_{\mathbf{x}} \mathrm{e}^{-i \mathbf{p} \cdot \mathbf{x}} \cdot\left\langle Q^{\dagger}\left(\mathbf{x}, t \mid \mathbf{0}, t_{s}, \xi_{j}\right)\left(\gamma_{5} \gamma_{3}\right) W\left(\mathbf{x}, t\left|\mathbf{p}, t_{s}\right| \mathbf{0}, t_{s}, \xi_{j}\right)\right\rangle .
\end{aligned}
$$

In this work we set the source at $t_{s}=4$ and impose the Dirichlet boundary condition in the time direction. We calculate the $Q$-type propagators for four sets of $\mathbf{q}$ and the $U(1)$ noise in (2.6) : $(\mathbf{q}$, noise $)=\{(\mathbf{0}, \xi),(\mathbf{0}, \eta),(\mathbf{p}, \xi),(-\mathbf{p}, \xi)\}$. The $W$-type propagators are calculated for 22 sets of $\mathbf{k}, t_{1}$ and $\mathbf{q}$ in $(2.10):\left(\mathbf{k}, t_{1} \mid \mathbf{q}\right)=\left\{\left(\mathbf{p}, t_{s} \mid \mathbf{0}\right),\left(-\mathbf{p}, t_{s} \mid \mathbf{0}\right),\left(\mathbf{0}, t_{s} \mid \mathbf{p}\right),\left(\mathbf{0}, t_{s} \mid-\mathbf{p}\right),\left(\mathbf{0}, t_{1}=4-21 \mid \mathbf{0}\right)\right\}$, with the same $U(1)$ noise $\xi$. All diagrams for the time correlation function can be calculated with combinations of these propagators. We choose $N_{R}=10$ for the number of $U(1)$ noise. We carry out additional measurements to reduce statistical errors using the source operator is located at $t_{s}+T / 2$ and the Dirichlet boundary condition is imposed at $T / 2$. We average over the two measurements for the analysis. Thus we calculate 520 quark propagators for each configuration. The total number of configurations analyzed are 800 separated by 5 trajectories [5].

\section{Results}

In Fig. 1 we plot the real part of the diagonal components $(\pi \pi \rightarrow \pi \pi$ and $\rho \rightarrow \rho)$ and the imaginary part of the off-diagonal components $(\pi \pi \rightarrow \rho, \rho \rightarrow \pi \pi)$ of $G(t)$. Our construction of 

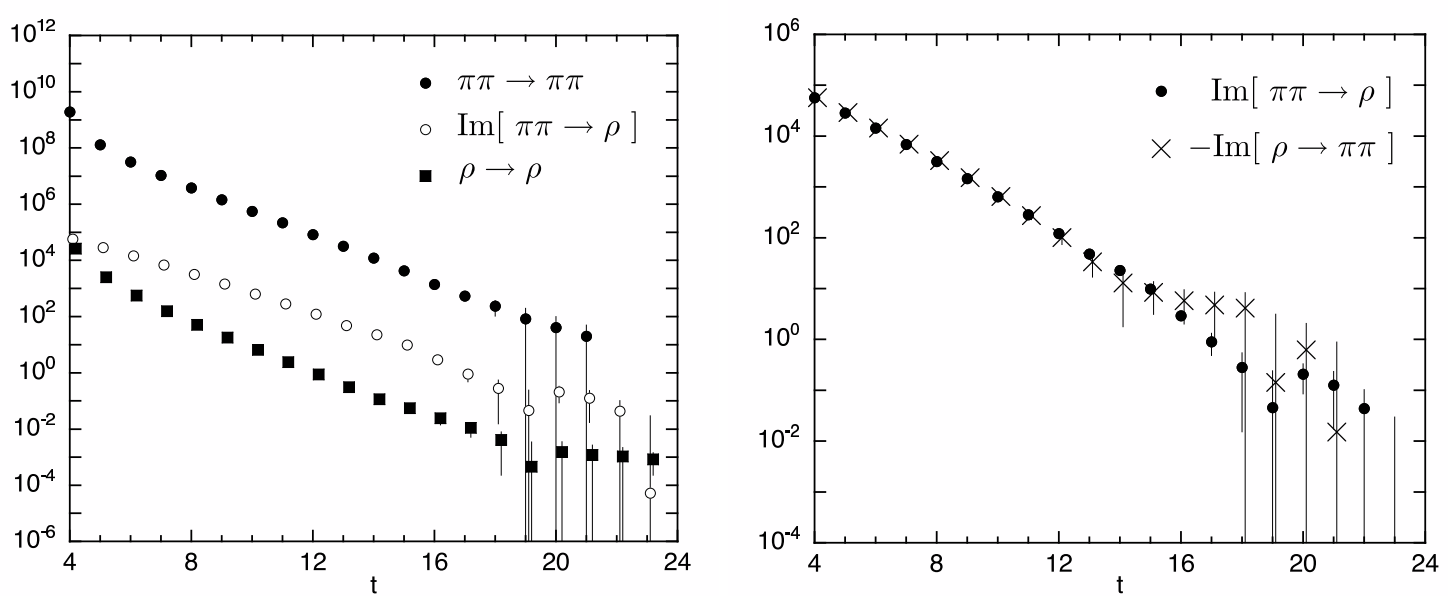

Figure 1: $G(t)$
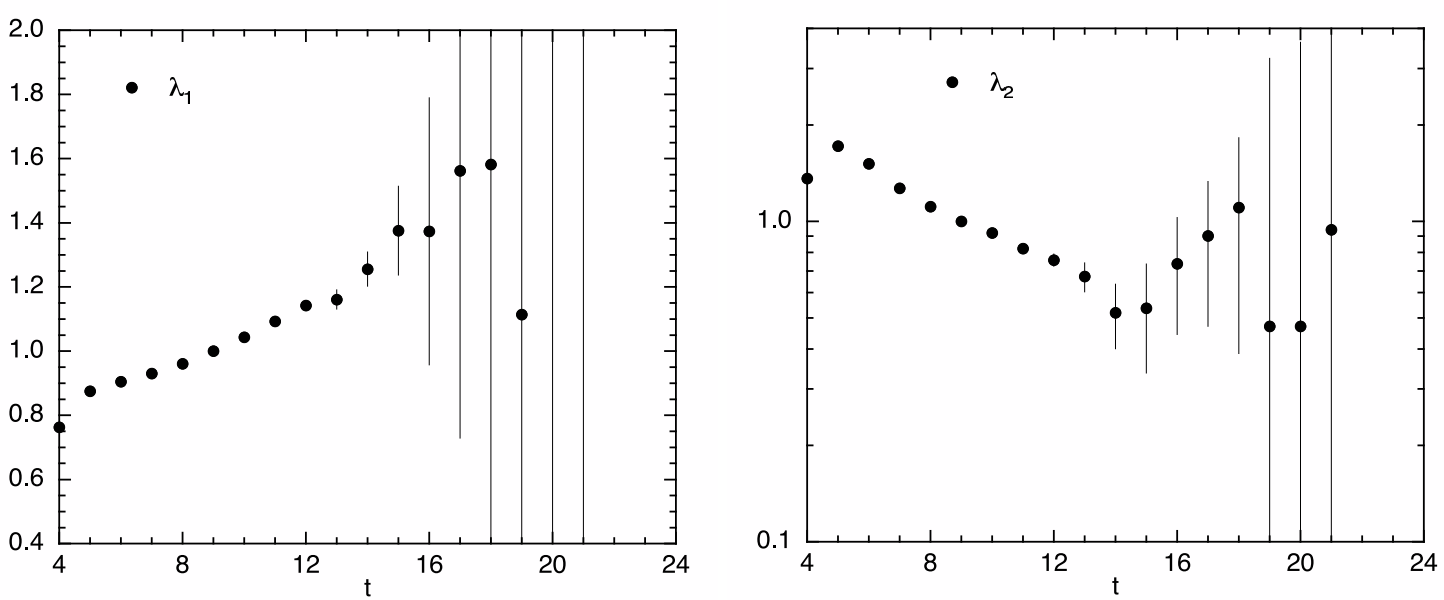

Figure 2: Normalized eigenvalues $\lambda_{1}\left(t, t_{R}\right)$ and $\lambda_{2}\left(t, t_{R}\right)$.

$G(t)$ is such that the sink and source operators are identical for a sufficiently large number of the $U(1)$ noise. In this case we can prove that $G(t)$ is an Hermitian matrix and the off-diagonal parts are pure imaginary from $P$ and $C P$ symmetry. We find that this is valid within statistics, but the statistical errors of the $\rho \rightarrow \pi \pi$ component is larger than those of $\pi \pi \rightarrow \rho$ in Fig. 1. In the following analysis we substitute $\rho \rightarrow \pi \pi$ by $\pi \pi \rightarrow \rho$ to reduce the statistical error.

The two eigenvalues $\lambda_{1}\left(t, t_{R}\right)$ and $\lambda_{2}\left(t, t_{R}\right)$ for the matrix $M\left(t, t_{R}\right)=G(t) G^{-1}\left(t_{R}\right)$ are shown in Fig. 2. We set the reference time $t_{R}=9$ and normalize the eigenvalues by the correlation function for the free two-pion system, $\left\langle 0\left|\pi(-\mathbf{p}, t) \pi\left(\mathbf{p}, t_{s}\right)\right| 0\right\rangle\left\langle 0\left|\pi(\mathbf{0}, t) \pi\left(\mathbf{0}, t_{s}\right)\right| 0\right\rangle$. Thus the slope of the figure corresponds to the energy difference $\Delta E_{n}=E_{n}-E_{1}^{0}$. We observe that the energy difference for $\lambda_{1}$ is negative and that for $\lambda_{2}$ is positive. This means that the two-pion scatting phase shift is positive for the lowest state and negative for the next higher state.

We extract the energy difference $\Delta E_{n}$ for both states by a single exponential fitting of the normalized eigenvalues $\lambda_{1}$ and $\lambda_{2}$ for the time range $t=10-16$. Then we reconstruct the energy $E_{n}$ in the moving frame by adding the energy of the two free pions, i.e., $E_{n}=\Delta E_{n}+E_{1}^{0}$, and convert it 


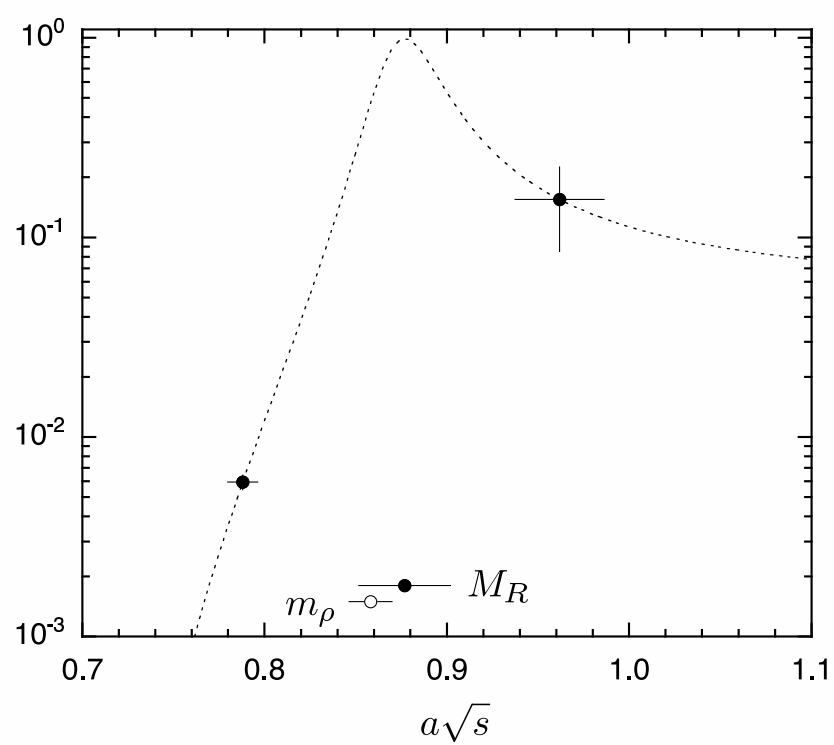

Figure 3: $\sin ^{2} \delta(\sqrt{s})$, position of $m_{\rho}$ and resonance mass $M_{R}$.

to the invariant mass $\sqrt{s}$. Substituting $\sqrt{s}$ into the Rummukainen-Gottlieb formula (2.1) we obtain the scattering phase shift :

\begin{tabular}{lc}
\hline \hline$a \sqrt{s}$ & $\tan \delta(\sqrt{s})$ \\
\hline $0.7880 \pm 0.0082$ & $0.0773 \pm 0.0033$ \\
$0.962 \pm 0.024$ & $-0.43 \pm 0.12$ \\
\hline \hline
\end{tabular}

The $\rho$ meson mass obtained at zero momentum is $a m_{\rho}=0.858 \pm 0.012$. Hence the sign of the scattering phase shifts at $\sqrt{s}<m_{\rho}$ is positive (attractive interaction) and that at $\sqrt{s}>m_{\rho}$ is negative (repulsive interaction) as expected. The corresponding results for $\sin ^{2} \delta(\sqrt{s})$, which is proportional to the scattering cross section of the two-pion system, are plotted in Fig. 3 together with the position of $m_{\rho}$.

In order to estimate the $\rho$ meson decay width at the physical quark mass we parameterize the scattering phase shift by the effective $\rho \rightarrow \pi \pi$ coupling constant $g_{\rho \pi \pi}$,

$$
\tan \delta(\sqrt{s})=\frac{g_{\rho \pi \pi}^{2}}{6 \pi} \cdot \frac{k^{3}}{\sqrt{s}\left(M_{R}^{2}-s\right)},
$$

with $g_{\rho \pi \pi}$ defined by the effective Lagrangian,

$$
L_{\text {eff. }}=g_{\rho \pi \pi} \cdot \varepsilon_{a b c}\left(k_{1}-k_{2}\right)_{\mu} \rho_{\mu}^{a}(p) \pi^{b}\left(k_{1}\right) \pi^{c}\left(k_{2}\right),
$$

where $k$ is the scattering momentum and $M_{R}$ is the resonance mass. The coupling $g_{\rho \pi \pi}$ generally depends on the quark mass and the energy, but our present data at a single quark mass do not provide this information. Here we assume that these dependences are small and try to estimate $g_{\rho \pi \pi}$ and $M_{R}$ from our results in (3.1). We also estimate the $\rho$ meson decay width at the physical 
quark mass from

$$
\Gamma_{\rho}=\frac{g_{\rho \pi \pi}^{2}}{6 \pi} \cdot \frac{\bar{k}_{\rho}^{3}}{\bar{m}_{\rho}^{2}}=g_{\rho \pi \pi}^{2} \times 4.128 \mathrm{MeV}
$$

where $\bar{m}_{\rho}$ is the $\rho$ meson mass at the physical quark mass and $\bar{k}_{\rho}$ is the scattering momentum at $\sqrt{s}=\bar{m}_{\rho}$.

Our final results are as follows.

$$
\begin{aligned}
a M_{R} & =0.877 \pm 0.025 \\
g_{\rho \pi \pi} & =6.01 \pm 0.63 \\
\Gamma_{\rho} & =149 \pm 31 \mathrm{MeV} .
\end{aligned}
$$

The resonance mass $M_{R}$ obtained from the scattering phase shift is consistent with $a m_{\rho}=0.858 \pm$ 0.012 obtained from the $\rho$ meson with zero momentum. The $\rho$ meson decay width $\Gamma_{\rho}$ at the physical quark mass is consistent with experiment $(150 \mathrm{MeV})$. In Fig. 3 we indicate the position of $M_{R}$ and draw the line given by (3.2) with $g_{\rho \pi \pi}$ and $M_{R}$ in (3.5).

\section{Summary}

We have shown that a direct calculation of the $\rho$ meson decay width from the scattering phase shift for the $I=1$ two-pion system is possible with present computing resources. However, several issues remain which should be investigated in future work. The most important issue is a proper evaluation of the quark mass and energy dependence of the effective $\rho \rightarrow \pi \pi$ coupling constant $g_{\rho \pi \pi}$. This constant is used to obtain the physical decay width at $m_{\pi} / m_{\rho}=0.18$ from our results at $m_{\pi} / m_{\rho}=0.41$ by a long chiral extrapolation. In principle we can estimate the decay width from the scattering phase shift without such a parameterization, if we have data for several energy values at or near the physical quark mass. This will be our goal toward the lattice determination of the $\rho$ meson decay.

This work is supported in part by Grants-in-Aid of the Ministry of Education (Nos. 17340066, 16540228, 18104005, 17540259, 13135216, 18540250, 15540251, 13135204, 16740147,18740139 ). The numerical calculations have been carried out on VPP5000/80 at Academic Computing and Communications Center of University of Tsukuba.

\section{References}

[1] S. Gottlieb, P.B. Mackenzie, H.B. Thacker, and D. Weingarten, Phys. Lett. B134 (1984) 346.

[2] R.D. Loft and T.A. DeGrand, Phys. Rev. D39 (1989) 2692.

[3] UKQCD Collaboration, C. McNeile and C. Michael, Phys. Lett. B556 (2003) 177.

[4] K. Rummukainen and S. Gottlieb, Nucl. Phys. B450 (1995) 397.

[5] CP-PACS Collaboration, Y. Namekawa et al., Phys. Rev. D70 (2004) 074503.

[6] M. Lüscher, Commun. Math. Phys. 105 (1986) 153; Nucl. Phys. B354 (1991) 531.

[7] CP-PACS Collaboration, T. Yamazaki et al., Phys. Rev. D70 (2004) 074513.

[8] M. Lüscher and U. Wolff, Nucl. Phys. B339 (1990) 222. 